\title{
WHAT ARE THE IMPACTS OF CLUSTER POLICIES?
}

\section{THE GERMAN EXPERIENCE}

\section{Michael Rothgang (RWI), Bernhard Lageman, Anne-Marie Scholz (ISG)*}

\section{Starting point}

Cluster policies in Germany

Cluster policies have changed structural policies in Germany in a
substantial and sustainable manner in the past decades:

1. All State Governments use cluster promotion to promote regional growth and innovation, while the Federal Government implemented several own cluster programmes.

2. Cluster policies are rather popular due to (i) a sound theoretical basis in cluster literature, (ii) flexibility of the instrument with cluster organizations as intermediaries that take up firm needs.

3. There are substantial differences between programs in respect to funding and targets.

To improve cluster policies it is important to understand the mechanisms of their policy success and failure.

Theory-based expectations

Cluster policies should

- promote existing clusters and function as midwife of emerging clusters,

encourage cooperation between firms, academia and state authorities,

increase the developmental potential of regional economies and promote technology evolution.

However...

- Evidence on the actual effects of cluster policy is limited,

- The experiences with practical cluster policy in Germany have not been assessed in a systematic manner yet.

\section{Research Questions}

How does the diversity of practical cluster policies relate to their intended effects?

2. To what extent and on what methodological basis were the Federal and State cluster programs evaluated?

3. What effects were identified and which questions remain open in respect to (a) the impact of cluster policies and (b) the evaluation methods applied?

\section{Evaluation studies}

\section{Availability and methods}

- Many official evaluation studies, however mostly ex ante or accompanying evaluations (some ex post evaluations mainly of Federal Programmes),

In many cases simple qualitative assessments, based on a limited set of indicators.

- We find a small number of more in-depth cluster policy impact analyses in the research literature (some qualitative, some quantitative).

Challenges

Most programme effects are the result of complex effect mechanisms with many external influences.

Politicians' interest in short-term results contradicts basic structural features of these programmes.

- Major policy effects can only become visible after longer timelags and based on a resource-consuming thorough analysis.

\section{Empirical Approach}

Qualitative analysis of the evidence on characteristics of German cluster policies, their intended effects, and the available evaluation studies; three components are combined:

- Cluster initiatives: Internet-based survey of 600 cluster initiatives from which 370 focus on innovation and knowledge transfer, deeper analysis of 30 randomly chosen technology-oriented cluster initiatives,

- Cluster policies: Survey of about 25 cluster programmes of the Federal Government and the 16 State Governments,

- Evaluation studies: Analysis of the available cluster literature (formal evaluations, empirical studies) on German cluster policy.

\section{Findings}

1. Cluster policies (and the related concept of network promotion) embrace a large range of different instruments from financial support of common activities to R\&D funding of projects related to a common cluster mission

2. Cluster initiatives are the intermediate organizations needed to promote cluster development; based on different characteristics, four types of cluster initiatives could be identified which relate to different intended policy effects.

Types of Cluster Initiatives, Characteristics, and Effects



3. Several evaluation studies have been conducted both at the Länder level and for Federal programmes. These evaluations were rather different in respect to which policy effects could be assessed. 3 Major results are:

Qualitative studies show that the policies were successful in shaping supportive environments for cluster actors and in offering assistance for existing and emerging R\&D cooperation networks. ${ }^{4}$

These activities were successful only in a part of the cases; there is more relevant knowledge on effects on the cluster than on the programme level.

- Success on cluster level was determined by multiple factors, including program design, financial endowment, abilities of the cluster management, but also the individual constellation of the cluster/cluster initiative.

Quantitative analyses identified effects for individual firms' input and output indicators. However, doubts arise with respect to the causal interpretation of some of the output-related results because of the probable time structure of impact patterns. ${ }^{5}$

4. While primary effects (induced activities and network development) could be clearly identified, much less is known about secondary policy effects (such as resulting inventions and innovations on the firm level, and impact on cluster population and performance on the cluster level).6

Evidence on Cluster Policy Impact from an Evolutionary Perspective

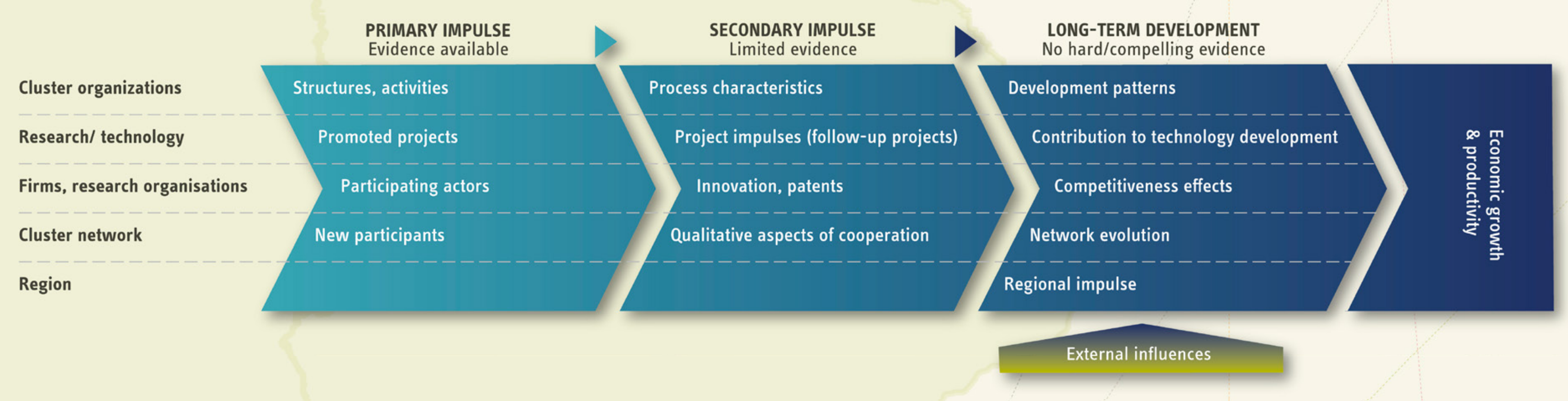

\section{Implications}

To gain more relevant insights into the long-term impact of cluster policy, impact research should reconstruct chains of effects of policy-induced cluster development and confront the findings with the original narratives of policy makers on expected cluster evolution.

2. New developments in the evaluation methodology of cluster policies are needed: improved qualitative approaches to understand the sequential effects of policy impulses (e.g. process tracing), the thoughtful application of advanced quantitative approaches based on theoretical impact 